\title{
Predictors of Negative Cone Biopsies and Its Clinical Significance
}

\author{
Beatriz Navarro Santana*, Raquel Sanz Baro \\ Obstetrics and Gynecology Department, Fundación Jiménez Díaz Hospital, Madrid, Spain \\ Email: *bea_0904@hotmail.com
}

How to cite this paper: Santana, B.N. and Baro, R.S. (2017) Predictors of Negative Cone Biopsies and Its Clinical Significance. Open Journal of Obstetrics and Gynecology, 7, 1092-1101.

https://doi.org/10.4236/ojog.2017.711110

Received: August 31, 2017

Accepted: October 16, 2017

Published: October 19, 2017

Copyright (c) 2017 by authors and Scientific Research Publishing Inc. This work is licensed under the Creative Commons Attribution International License (CC BY 4.0).

http://creativecommons.org/licenses/by/4.0/ (c) (i) Open Access

\begin{abstract}
Objective: To determine the predictors of absence of lesion on cone biopsy (white cone). We evaluated several factors including parity, cytology, human papillomavirus (HPV) typing, biopsy, colposcopy, smoking habit, use of oral contraceptive or condom, and immunosuppression as predictors for absence of dysplasia. Methods: Of 510 patients with CIN (cervical intraepithelial neoplasia) treated by loop electrosurgical excision (LEEP) in the Fundación Jimenez Diaz hospital between 2012 and 2016, 51 (10\%) patients had a white cone and were defined as the study group. The control group was established from two randomly selected controls for each case $(n=102)$. Results: The study group had a higher prevalence of low-grade cytology ( $<<0.001)$, minor changes $(\mathrm{p}<0.01)$, and CIN $2(<0.001)$ than the control group. The study group had a lower rate of oral contraceptive use $(\mathrm{p}=0.005)$, as demonstrated by the multivariate analysis (except for low-grade cytology). Conclusions: In conclusion, this study indicates that the incidence of white cone in our institution is $10 \%$ of all the cone biopsy and women with low-risk cytology, minor changes in colposcopy, CIN 2, and no use of oral contraceptive have a high probability of having no lesions in the conization specimen.
\end{abstract}

\section{Keywords}

White Cone, Cervical Intraepithelial Dysplasia, Predictors, Study Group, Control Group

\section{Introduction}

Cervical intraepithelial neoplasia (CIN) is a premalignant condition of the cervix. The term CIN refers to squamous abnormalities. Cervical glandular neoplasia comprises adenocarcinoma in situ and adenocarcinoma. CIN can be low- or high-grade. Low-grade lesions have low rates of progression to carcinoma [1]. The 
terminology used to describe cervical lesions has varied over the years. Until recently it was based on the Bethesda system [2] [3] [4] with different names given to findings on cytology and biopsy. Thus, findings on cytologic examination were named SIL (squamous intraepithelial lesion) and biopsy findings were named CIN (cervical intraepithelial neoplasia) according to three different degrees of severity. In 2012, the LAST system (Lower Anogenital Squamous Terminology) used the same terminology to report cytologic and histologic findings [5]. The annual incidence of CIN in USA is $4 \%$ for CIN 1 and $5 \%$ for CIN stage [2] [3] [6]. The treatment is based on the results of the biopsy under colposcopic examination. These examinations may be excisional (cervical conization) or ablative. Excisional treatments have diagnostic and therapeutic targets and are usually reserved for high-grade lesions with histologic confirmation or in case of cytologic-histologic discrepancy. These are also used for low-risk lesions that persist for more than two years, and are large, or are located in the endocervical canal [7] [8]. Despite these strict criteria, cone specimens without residual CIN have been documented. For this reason, several studies have attempted to elucidate the predictors of absence of cervical neoplasia in the cone specimen, as well as their clinical significance and incidence [9]-[16].

An incidence from $13 \%$ to $18 \%$ of white cone has been estimated after a cone biopsy in the literature [9] [10] [11] [12] [13] [16]. There are many variables that can predict absence of CIN in the cone specimen, though a low viral load $(\leq 10$ RLU) has the strongest association [11]. However, white cone does not imply the absence of recurrent/persistent disease during follow-up, and therefore postsurgical management must be the same as in other cone biopsies.

In this study, we analyzed which clinical, cytologic, histologic, colposcopic or virologic factors predicted white cone biopsy in case of previous high-grade biopsy. We also analyzed the impact of white cone biopsy in terms of persistence/recurrence of disease in daily practice.

\section{Methods}

\subsection{Case Selection of Cone Biopsy}

Five hundred and ten cone biopsies were carried out in the department of gynaecology and obstetrics of the Fundación Jimenez Diaz University Hospital. These procedures were performed from January 2012 to February 2016 by five different gynaecologists from the lower genital-tract pathology department, each having the same level of training. All patients were previously referred to the department due to abnormal cytology. HPV testing and colposcopy were subsequently performed with a guided biopsy if these tests were indicated or endocervical curettage if the transformation zone was not visible or was partially visible.

The study was designed as a retrospective case control analysis. The study group included patients with a negative histology in the conization specimen ( $\mathrm{n}$ $=51$ ) and CIN stage $2-3$ on previous colposcopy-guided biopsy. We included two randomly selected controls for each case; therefore, 102 controls were se- 
lected. These controls also had a previous colposcopy-guided biopsy informed as CIN 2 - 3, and a high-grade cervical dysplasia confirmed the conization specimen.

The study was conducted according to the principles of the Declaration of Helsinki. Written informed consents were obtained from all patients before the cone biopsy.

According to the recommendations of the American Society for Colposcopy and Cervical Pathology [17], criteria for cone biopsy of patients with HSIL/CIN 2 - 3 were: 1) a histological diagnosis of CIN 2 - 3 by colposcopy-guided biopsy or endocervical curettage; or 2) two Pap tests defined as HSIL separated by six months and excluding vaginal lesions on colposcopy.

\subsection{Cytology, HPV Testing, Colposcopy, and Cone Biopsy}

We collected data related to parity, age, months from diagnosis to surgery, previous cytology and HPV type, previous biopsy, colposcopy findings, smoking habit, immunosuppression, and condom and hormonal contraceptive use.

We used the Thinprep Test ${ }^{\circledast}$ liquid-based Pap testing and DNA hybrid capture for 13 types of high risk HPV detection (Qiagen HC2 High-Risk HPV-DNA Test ${ }^{\circledast}$ technology Hybrid Capture $2^{\circledR}$ ), taking two independent samples with a cytobrush for cytology and HPV detection (DNA pap cervical sampler ${ }^{\oplus}$ ).

With respect to the results of the cytology study, we grouped data into low-grade intraepithelial lesions (ASCUS and LSIL) and high-grade lesions (HSIL, ASC-H). The results of HPV testing were grouped as negative, presence of HPV 16/18 infection, and presence of other high-risk HPV types other than $16 / 18$.

Colposcopy was conducted using an Optomic OP-C5 colposcope after preparing the cervix with 5\% acetic acid and Lugol's iodine. The location of the lesions found during colposcopy was described according to clock-face method. Lesion size was established depending on the number of quadrants that the lesion covered. A colposcopy was defined as satisfactory if the transformation zone was entirely visible. The 2011 colposcopic terminology of the International Federation for Cervical Pathology and Colposcopy groups the lesions into normal findings, abnormal colposcopic findings, or findings suspicious for invasion [18]:

- Lesions suggestive of low-grade disease (grade-1 findings): thin acetowhite epithelium; irregular; geographic border; fine mosaic; fine punctuation;

- Lesions suggestive of high-grade disease (grade-2 findings): dense acetowhite epithelium, rapid appearance of ace to whitening, cuffed crypt (gland openings), coarse mosaic, coarse punctuation, sharp border, inner border sign, and ridge sign;

- Lesions suggestive of invasive cancer: atypical vessels, fragile vessels, irregular surface, exophytic lesion, necrosis, ulceration (necrotic), tumor/gross neoplasm; and Cervical biopsies were done using the Kervokian cervical biopsy forceps, which removed approximately $1-2 \mathrm{~mm}$ of tissue. Local anaesthesia 
was not used.

Cone biopsies were performed using LEEP (loop electrosurgical excision procedure). First, we used a metal speculum connected to an aspirator for smoke evacuation. Second, we dyed the cervix with acetic acid and lugol's iodine to delimit the lesions. Third, we injected $1 \%$ lidocaine or mepivacaine into the four quadrants of the cervix and paracervix. The loop size depended on the size of the cervix: we used LEEP loop electrodes of a medium radius $(1.0-1.5 \mathrm{~cm})$ and LEEP loop electrodes of a large radius $(2.0-2.5 \mathrm{~cm})$ manufactured by Cooper Surgical ${ }^{\oplus}$. After the procedure, we achieved haemostasis with a ball electrode by Cooper Surgical $^{\oplus}$. Finally, we marked the cone with a long stitch at $12 \mathrm{~h}$.

We discharged the patients on the same day of the procedure and recommended that they return to the emergency department in case of heavy bleeding, severe abdominal pain, or fever higher than $38^{\circ} \mathrm{C}$.

\subsection{Follow-Up}

The first post-treatment control was performed in all patients who at six months had free margins in cone biopsy and consisted of cytology, high-risk HPV infection test, and colposcopy and biopsy if required. If there weren't affected margins and no indication of new conization or hysterectomy, we performed the first control 3 - 4 months after surgery. In each patient, we performed a liquid Pap test with the ThinPrep ${ }^{\otimes}$ system and in vitro DNA hybridization assay to detect 13 types of high-risk HPV (Qiagen HC2 High-Risk HPV DNA Test ${ }^{\circledR}$ technology Hybrid Capture $2^{\circledast}$ ) taking 2 independent samples for cytology and HPV detection (DNA Pap cervical sampler ${ }^{\circledast}$ ). Follow-up visits took place every six months for two years. Persistent/recurrent disease was defined as the presence of an abnormal cytology result, positive HPV testing with normal cytology, or biopsy with intraepithelial neoplasia. If all the results (cytology and HPV testing) were negative for two years, the patients were discharged and were referred to their primary-care physician for further follow-up.

\subsection{Statistical Analysis}

For statistical analysis, we used the program $\mathrm{R}$ version $3.1 .2^{\circledast}$. Quantitative variables were described using mean and standard deviation, and we used the absolute and relative frequencies ( $\mathrm{N}$ and \%) to describe qualitative variables. $\mathrm{Re}$ garding qualitative variables, we evaluated the association between the variable and the status of the study/control group using the chi-square test. In cases where chi-square testing was not appropriate, we used the Fisher exact test. Concerning quantitative variables, Mann-Whitney $U$ test was used for comparison of months between biopsy and surgery and Student $t$ test to compare ages. A p-value of 0.05 was considered statistically significant. We performed a multivariate approach using factors with $\mathrm{p}$-values $<0.2$. We used the risk estimation as odds ratio (OR) for each variable, with $95 \%$ confidence intervals and the p-value. 


\section{Results}

The mean age of the patients in the study group was $37.2 \pm 9.0$ years, and in the control group, the mean age was $34.2 \pm 7.2(\mathrm{p}=0.028)$. The mean number of months between diagnosis and surgery was $1.2 \pm 0.5$ and $1.5 \pm 0.9$ in the study and control groups, respectively ( $\mathrm{p}=0.023$ ).

We observed differences between the study and control groups in terms of low- and high-risk cytology, major and minor changes, CIN 2 and 3, use of hormonal contraceptive, and months from diagnosis to surgery. The study group had a higher prevalence of low-grade cytology $(\mathrm{p}<0.001)$, minor changes $(\mathrm{p}<0.01)$, and CIN $2(<0.001)$ than the control group. The study group had a lower prevalence of use of hormonal contraceptive $(\mathrm{p}=0.005)$.

No differences were observed between the two groups in terms of HPV type $(\mathrm{P}=0.411)$, size of the lesion which was divided in $1-2$ quadrants or $3-4$ quadrants, active smoking habit $(\mathrm{p}=0.467)$, condom use $(\mathrm{p}=0.867)$, parity $(\mathrm{p}=$ 0.806 ), immunosuppression ( $\mathrm{p}=1.00$ ), and age group. Though not reaching statistical significance, the prevalence of infection by HPV type 16/18 and the size of the lesion of $3-4$ quadrants were greater in the control than in the study group.

The median duration of follow-up in the study group and the control group was 15.4 and 17.8 months, respectively $(\mathrm{p}=0.113)$. Thus, there was no difference in terms of follow-up between the control and study group. Two of 51 (3.9\%) patients from the study group were lost to follow-up (Table 1 and Table 2).

No significant differences between the study and control group were observed in terms of recurrent/persistent disease after conization.

Table 3 shows the multivariate analysis, which is interpreted as follows:

- Major/minor changes in colposcopy: In this case, we compared major and minor changes. The estimated OR indicates that patients with major changes are less likely to have no lesion in the cone specimen than are patients with minor changes.

- Biopsy: In this case, we compared between CIN 3 vs. CIN 2. The estimated OR indicates that patients with CIN 3 are less likely to have no lesion in the cone specimen than are patients with CIN 2.

- Hormonal contraceptives: In this case, we performed a comparison between patients who take hormonal contraceptives and patients who don't. The estimated OR indicates that the consumers' patients are less likely to have no lesion in the cone specimen than are the no consumer patients.

- Period between biopsy and conization: The estimated OR indicates that patients with a long period between biopsy and conization have a decreased probability of having no lesion in the cone specimen.

\section{Discussion}

CIN is a premalignant condition of the cervix and the most common disease affecting the female genital tract. HPV is the only necessary factor for the 
Table 1. Comparisons between the control and the study group in terms of qualitative characteristics.

\begin{tabular}{|c|c|c|c|}
\hline & Control group & Study group & $\mathrm{p}$ \\
\hline Previous cytology & & & $<0.001$ \\
\hline Low grade & $42(44.7 \%)$ & $39(78.0 \%)$ & \\
\hline High grade & $52(55.3 \%)$ & $11(22.0 \%)$ & \\
\hline Previous HPV & & & 0.411 \\
\hline Others & $30(30.6 \%)$ & $18(39.1 \%)$ & \\
\hline $16,18,45$ & $68(69.4 \%)$ & $28(60.9 \%)$ & \\
\hline Lesion size & & & 0.102 \\
\hline $1-2$ quadrants & $82(82.8 \%)$ & $47(94.0 \%)$ & \\
\hline 3 - 4 quadrants & $17(17.2 \%)$ & $3(6.0 \%)$ & \\
\hline Colposcopy findings & & & $<0.001$ \\
\hline Major changes & $50(49.0 \%)$ & $41(82.0 \%)$ & \\
\hline Minor changes & $52(51.0 \%)$ & $9(18.0 \%)$ & \\
\hline Previous biopsy & & & $<0.001$ \\
\hline CIN 2 & $16(15.5 \%)$ & $32(61.5 \%)$ & \\
\hline CIN 3 & $87(84.5 \%)$ & $20(38.5 \%)$ & \\
\hline Smoking habit & & & 0.467 \\
\hline Yes & $27(31.0 \%)$ & $19(38.8 \%)$ & \\
\hline No & $60(69.0 \%)$ & $30(61.2 \%)$ & \\
\hline Condon use & & & 0.867 \\
\hline Yes & $50(53.2 \%)$ & $27(56.2 \%)$ & \\
\hline No & $44(46.8 \%)$ & $21(43.8 \%)$ & \\
\hline Hormonal contraceptive & & & 0.005 \\
\hline Yes & $30(37.0 \%)$ & $6(12.5 \%)$ & \\
\hline No & $51(63.0 \%)$ & $42(87.5 \%)$ & \\
\hline Parity & & & 0.806 \\
\hline Nulliparous/Miscarriage & $70(70.7 \%)$ & $35(67.3 \%)$ & \\
\hline Pregnancy & $29(29.3 \%)$ & $17(32.7 \%)$ & \\
\hline Immunosuppression & & & 1.000 \\
\hline Yes & $2(1.9 \%)$ & $1(1.9 \%)$ & \\
\hline No & $101(98.1 \%)$ & $51(98.1 \%)$ & \\
\hline Age & & & 0.483 \\
\hline$\leq 35$ & $63(61.2 \%)$ & $28(53.8 \%)$ & \\
\hline$>35$ & $40(38.8 \%)$ & $24(46.2 \%)$ & \\
\hline Age & & & 0.187 \\
\hline$\leq 40$ & $86(83.5 \%)$ & $38(73.1 \%)$ & \\
\hline$>40$ & $17(16.5)$ & $14(26.9 \%)$ & \\
\hline Age & & & 0.098 \\
\hline$\leq 50$ & $101(98.1)$ & $48(92.3 \%)$ & \\
\hline$>50$ & $2(1.9)$ & $4(7.7 \%)$ & \\
\hline Cytology 6 months & & & 1.000 \\
\hline Negative & $97(96.0 \%)$ & $50(96.2 \%)$ & \\
\hline Positive & $4(4.0 \%)$ & $2(3.8 \%)$ & \\
\hline
\end{tabular}


Table 2. Comparisons between the control and the study group in terms of quantitative characteristics.

\begin{tabular}{cccc}
\hline Variable & Control group & Study group & p value \\
\hline Age & $34.2 \pm 7.2$ & $37.2 \pm 9.0$ & 0.028 \\
Months from diagnosis to surgery & $1.5 \pm 0.9$ & $1.2 \pm 0.5$ & 0.023 \\
\hline
\end{tabular}

Table 3. Multivariate analysis.

\begin{tabular}{ccc}
\hline Variable & OR & (IC 95\%) \\
\hline Major Changes & 0.329 & $(0.115-0.873)$ \\
Previous biopsy (CIN 3) & 0.102 & $(0.036-0.262)$ \\
Hormonal contraceptive & 0.186 & $(0.050-0.575)$ \\
Months diagnosis-surgery & 0.414 & $(0.172-0.875)$ \\
\hline
\end{tabular}

development of CIN, although not a sufficient condition to cause the disease [19]. The two main factors associated with the development of high-grade CIN are virus subtype and virus persistence: HPV 16 and 18 are the most frequently associated with $\mathrm{CIN} 2+$, disease persistence, and progression to invasive cancer. Loop electrosurgical excision procedure has become an effective treatment for cervical dysplasia. However, the complete absence of dysplasia in the conization specimen after a high grade cervical biopsy is a phenomenon that has been documented in very few studies and occurred in $10 \%$ of cases at our institution. This percentage is similar to other studies: $13.8 \%$ in the study by Livasy et al. [9], $17.7 \%$ in the series by Ryu et al. [10], 16\% in the series by Rodriguez-Manfredi et al. [11], 10\% in the study by Witt et al. [12], 17\% by Carrig et al. [13], and $18.2 \%$ in the study of Nam et al. [16].

In our study, patients with low-risk cytology, minor changes in colposcopy, CIN 2, and absence of oral contraceptive use had an increased probability of having a white cone. However, we did not observe differences between the groups in terms of HPV type, size of the lesion, active smoking habit, condom use, parity, immunosuppression, age. Despite not being statistically significant, the percentage of infection by HPV type 16/18 and lesion with a size of $3-4$ quadrants was greater in the control than in the study group.

In the study by Manfredi et al., a negative hr-HPV test result, a low viral load, negative cytology results, LSIL in the preconization cytology, CIN1 in the preconization biopsy, and minor changes and lesion size $\leq 1$ quadrant in the preconization colposcopy were statistically significant.

In the series by Ryu et al., only low HPV load was significantly associated with the probability of absence of a lesion in the conization specimen [10].

In the study by Nam et al., CIN 2, a low HPV viral load, and HPV infection other than type 16 or absence of high-risk HPV infection were associated with absence of lesions in LEEP specimens [16].

According to previous studies by Ryu et al. [10], Rodriguez-Manfred et al. 
[11], and Nam et al. [16], a low viral load was related to the absence of dysplasia in cone specimens. In our institution, viral load readings before the cone biopsy were not available.

In the series by Nam et al., HPV infection by a virus type other than 16 was significantly related to the presence of a white cone biopsy, possibly because HPV-16 infection causes larger lesions [20]. In our series no differences were observed between the groups in terms of HPV type, though there was a major percentage of HPV 16/18 infection in the control group.

Different causes have been hypothesized to explain the absence of dysplasia in the conization specimen. First, the CIN is removed completely during the biopsy because it is small [10]. In the study by Rodriguez-Manfredi [11], patients with lesions involving less than one quadrant of the cervix had an increased probability of having a white cone biopsy. Second, the lesion can regress spontaneously, and a regression of $20 \%$ has been documented in cases of CIN 2 - 3 [21]. Third, a pathologist may not have been capable of observing the area of the lesion [10], especially if they are not experienced pathologists. Finally, CINs are missed and not removed by LEEP [10]; this possibility is very unlikely because conization must include the transformation zone, and all lesions are located in this area [11].

Many factors have been associated with persistent/recurrent disease after cone biopsy: positive margins, endocervical gland involvement by dysplasia and multifocal disease [9], HPV-16 type [22], high viral load [10] [11] age, and parity. However, absence of dysplasia in the cone specimen is not a predictor of recurrence [9]-[16].

In our study, after six months of follow-up, $96.2 \%$ of patients in the study group had a negative cytology and 53\% had a negative high-risk HPV test, while $96 \%$ of patients in the control group had a negative cytology and $94.1 \%$ had a negative high-risk HPV test. There was no statistical difference between the two groups in terms of recurrence/persistence of the disease. These results are similar to other studies [9]-[16]. White cone biopsy does not ensure the absence of recurrent disease [16], so these patients need close-follow up as other patients who have undergone cone biopsy [10] [16].

\section{Conclusions}

In conclusion, this study indicates that the incidence of white cone in our institution is $10 \%$ of all the cone biopsy; this incidence is similar to the series by Livasy et al. [9], Ryu et al. [10], Rodriguez-Manfredi et al. [11], Witt et al. [12], Carrig et al. [13], and Nam et al. [16] This series also confirms that women with low-risk cytology, minor changes in colposcopy, CIN 2, and no use of oral contraceptive have a high probability of having no lesions in the conization specimen. Women with negative cone and women with residual lesions as evidenced by LEEP have similar rates of persistent/recurrent disease.

The limitations of our study are its retrospective nature and the fact that we did not have readings on viral load before and after the biopsy cone, as in our 
institution this is currently not available. On the other hand, our study has a good sample size and the diagnosis, cone biopsy, and follow up of patients were done by a small number of gynaecologists with a high level of training. We also had a detailed record of every single colposcopy image, and the pathologist who analysed the conization specimen was always the same person, making him an experienced pathologist.

\section{Conflict of Interest}

The authors declare that they do not have any conflicts of interest.

\section{References}

[1] Bansal, N., Wright, J.D., Cohen, C.J. and Herzog, T.J. (2008) Natural History of Established Low Grade Cervical Intraepithelial (CIN 1) Lesions. Anticancer Research, 28,1763 .

[2] Solomon, D., Davey, D., Kurman, R., et al. (2002) The 2001 Bethesda System: Terminology for Reporting Results of Cervical Cytology. JAMA, 287, 2114. https://doi.org/10.1001/jama.287.16.2114

[3] Luff, R.D. (1992) The Bethesda System for Reporting Cervical/Vaginal Cytologic Diagnoses. Report of the 1991 Bethesda Workshop. American Journal of Clinical Pathology, 98, 152. https://doi.org/10.1093/ajcp/98.2.152

[4] National Cancer Institute Workshop. (1989) The 1988 Bethesda System for Reporting Cervical/Vaginal Cytological Diagnoses. JAMA, 262, 931.

https://doi.org/10.1001/jama.1989.03430070079034

[5] Darragh, T.M., Colgan, T.J., Thomas Cox, J., et al. (2013) The Lower Anogenital Squamous Terminology Standardization Project for HPV-Associated Lesions: Background and Consensus Recommendations from the College of American Pathologists and the American Society for Colposcopy and Cervical Pathology. International Journal of Gynecological Pathology, 32, 76. https://doi.org/10.1097/PGP.0b013e31826916c7

[6] Insinga, R.P., Glass, A.G. and Rush, B.B. (2004) Diagnoses and Outcomes in Cervical Cancer Screening: A Population-Based Study. American Journal of Obstetrics \& Gynecology, 191, 105. https://doi.org/10.1016/j.ajog.2004.01.043

[7] ACOG Practice Bulletin Number 66. (2005) Management of Abnormal Cervical Cytology and Histology. Obstet Gynecol, 106, 645-664.

[8] Van Hamont, D., van Ham, M.A., Struik-van der Zanden, P.H., Keijser, K.G., Bulten, J., Melchers, W.J., et al. (2006) Long-Term Follow-Up after Large-Loop Excision of the Transformation Zone: Evaluation of 22 Years Treatment of High-Grade Cervical Intraepithelial Neoplasia. International Journal of Gynecological Cancer, 16, 615-619. https://doi.org/10.1111/j.1525-1438.2006.00404.x

[9] Livasy, C.A., Moore, D.T. and Van, L.L. (2004) The Clinical Significance of a Negative Loop Electrosurgical Cone Biopsy for High-Grade Dysplasia. Obstetrics \& Gynecology, 104, 250-254. https://doi.org/10.1097/01.AOG.0000132803.88049.84

[10] Ryu, A., Nam, K., Chung, S., Kim, J., Lee, H., Koh, E., et al. (2010) Absence of Dysplasia in the Excised Cervix by a Loop Electrosurgical Excision Procedure in the Treatment of Cervical Intraepithelial Neoplasia. Journal of Gynecologic Oncology, 21, 87-92. https://doi.org/10.3802/jgo.2010.21.2.87

[11] Rodriguez-Manfredi, A., Alonso, I., del Pino, M., Fusté, P., Torné, A. and Ordi, J. 
(2013) Predictors of Absence of Cervical Intraepithelial Neoplasia in the Conization Specimen. Gynecologic Oncology, 128, 271-276.

[12] Witt, B.L., Factor, R.E., Jarboe, E.A. and Layfield, L.J. (2012) Negative Loop Electrosurgical Cone Biopsy Finding Following a Biopsy Diagnosis of High-Grade Squamous Intraepithelial Lesion: Frequency and Clinical Significance. Archives of Pathology \& Laboratory Medicine, 136, 1259-1261. https://doi.org/10.5858/arpa.2011-0494-OA

[13] Carrigg, A., Teschendorf, C., Amaro, D., Weidner, N., Tipps, A., Shabaik, A., et al. (2013) Examination of Sources of Diagnostic Error Leading to Cervical Cone Biopsies with No Evidence of Dysplasia. American Journal of Clinical Pathology, 139, 422-427. https://doi.org/10.1309/AJCP6BSD0SNGQLHQ

[14] Golbang, P., Scurry, J., de Jong, S., McKenzie, D., Planner, R., Pyman, J., et al. (1997) Investigation of 100 Consecutive Negative Cone Biopsies. British Journal of Obstetrics and Gynaecology, 104, 100-104. https://doi.org/10.1111/j.1471-0528.1997.tb10658.x

[15] Koc, N., Sahin, D. and Ayas, S. (2013) Re-Evaluation of Negative Cone Biopsy Results after a Positive Cervical Biopsy Finding. Journal of Lower Genital Tract Disease, 17, 154-159.

[16] Nam, K., Ryu, A., Jeon, S., Kim, J., Kwak, J. and Park, B. (2015) Clinical Significance of a Negative Loop Electrosurgical Excision Procedure Biopsy in Patients with Biopsy-Confirmed High-Grade Cervical Intraepithelial Neoplasia. Journal of Lower Genital Tract Disease, 19, 103-109. https://doi.org/10.1097/LGT.0000000000000061

[17] Wright, T.C., Massad, L.S., Dunton, C.J., Spitzer, M., Wilkinson, E.J. and Solomon, D. (2006) Consensus Guidelines for the Management of Women with Cervical Intraepithelial Neoplasia or Adenocarcinoma in Situ. Journal of Lower Genital Tract Disease, 11, 223-239. https://doi.org/10.1097/LGT.0b013e318159408b

[18] Bornstein, J., Bentley, J., Bösze, P., et al. (2011) Colposcopic Terminology of the International Federation for Cervical Pathology and Colposcopy. Obstetrics \& Gynecology, 120, 166. https://doi.org/10.1097/AOG.0b013e318254f90c

[19] Castellsagué, X., Díaz, M., de San josé, S., et al. (2006) Worldwide Human Papillomavirus Etiology of Cervical Adenocarcinoma and Its Cofactors: Implications for Screening and Prevention. Journal of the National Cancer Institute, 98, 303. https://doi.org/10.1093/jnci/djj067

[20] Nam, K., Kwak, J., Kim, J. and Jeon, S. (2013) Human Papillomavirus Type 16 Causes Larger Colposcopic Lesions than Other HPV Types in Patients with Grade 3 Cervical Intraepithelial Neoplasia. Journal of Lower Genital Tract Disease, 17, 1-5. https://doi.org/10.1097/LGT.0b013e31825afd5b

[21] Trimble, C.L., Piantadosi, S., Gravitt, P., Ronnett, B., Pizer, E., Elko, A., et al. (2005) Spontaneous Regression of High-Grade Cervical Dysplasia: Effects of Human Papillomavirus Type and HLA Phenotype. Clinical Cancer Research, 11, 4717-4723. https://doi.org/10.1158/1078-0432.CCR-04-2599

[22] Gök, M., Coupé, V.M., Berkhof, J., Verheijen, R.H., Helmerhost, T.J., Hogewoning, C.J., et al. (2007) HPV16 and Increased Risk of Recurrence after Treatment for CIN. Gynecologic Oncology, 104, 273-275. 